\title{
Astronomy and Mathematics Education
}

\author{
Rosa M. Ros \\ Universidad Politecnica de Catalunya, Spain \\ E-mail: ros@mat.upc.es
}

Astronomy has an interdisciplinary aspect that in some cases is very positive. It is interesting to combine Astronomy with other topics and introduce Astronomy in general projects in school in order to integrate several courses, for instance Physics, Mathematics, Geography, Biology or History; however these kinds of projects are sporadic. In order to promote Astronomy it is better to try to introduce it as a course in its own right. The viability of this situation depends on the national curriculum and the plans of the Education Ministry in each country. In general, the majority of countries do not have Astronomy as a specific course and it is not easy to achieve. Therefore, if this option is not possible, we should adapt to the real situation, that is to say include Astronomy as a part of other courses. This contribution aims to propose a new point of view which could work to make this the case.

Commonly, Physics teachers include some Astronomy in their classes. In some cases it is possible that other Science teachers (biologists or geologists) or non-Science teachers (geographers or historians) also teach Astronomy. Of course, in order to promote Astronomy, we have to promote all these possibilities; however the author of this paper would like to introduce Astronomy content within Mathematics courses as well.

This idea has several advantages and very few disadvantages. If Mathematics teachers try to introduce Astronomy into their classes, the situation will be better for everyone, because Astronomy will be promoted, and mathematics enriched. The only obvious disadvantage is that, if the astronomical content or the mathematical application is too difficult, the effect could be counter-productive for both subjects; we have to be prudent, and choose the examples very carefully. The advantages are as follows:

- Mathematics is not very attractive to pupils; astronomy is more motivating. "Students are not interested in discovering what an ellipse is, but they are really concerned about the possibility of the Moon crashing into the Earth"

- Schools do not have Mathematics laboratories. Mathematics is a very theoretical subject for students and in general seems unconnected with the real world. Lay people think that Mathematics is necessary to count, but practically nothing more. Anything more than basic arithmetical rules seems beyond them. Astronomy can offer Mathematics teachers a laboratory: the school grounds. If it is not cloudy students can observe the sky, take measurements and analyze the results obtained with their mathematical knowledge (taking them beyond arithmetic) and it is 
also possible to introduce scientific methodology and study the margins of errors.

"Students are not very interested in calculating the third side of a triangle from the other sides and angles, but they will be interested in finding the distance between the Earth and the Moon after making their own observations. Maybe the results are not very accurate but the experience will be very motivating and they will never forget it".

- Mathematics appears from the first course in primary school until the end of secondary school. Students study Mathematics for many years, providing an enormous opportunity to introduce many different astronomical ideas. Whereas if Astronomy appears only in physics courses, it is introduced very late in the curriculum. It is, therefore, better to also introduce Astronomy in Mathematics courses.

"8-9 year-old students are very curious. They ask a lot of things about their surroundings. Why does the Moon look like a croissant? And, why sometimes not? Why can we see the Moon in the morning? (In children's stories the Moon only appears at night)".

We are currently missing the opportunity to harness the curiosity of these younger students because, to obtain answers, they have to wait until they study physics in secondary school.

\section{Conclusions}

It is very important to offer Mathematics teachers a good selection of topics and activities connecting Astronomy and Mathematics. If they have good materials they will use them. Of course it is important to select very carefully a collection of astronomical items interesting from a mathematical point of view, according with its curriculum and related to the real world, in order to attract the students. We have to make sure students and teachers are satisfied if we want to achieve success.

It is necessary to say that this option is not incompatible with teaching Astronomy in physics courses. The interests of Physics and Mathematics teachers are not the same, however; they can offer a complementary point of view.

Several examples of suitable materials for mathematics teachers follow:

- Angles and star trails (the same angle for the same exposure time in a photograph of the sky)

- Logarithms and visual magnitudes (students are surprised when they discover that their eyes know logarithms, and perhaps they failed the logarithms' exam in the Mathematics course!)

- Plane trigonometry related positions (related orbital movements)

- Spherical trigonometry and ecliptic obliquity (relationships on ecliptic obliquity and latitude)

- Conic curves related to sundials (by finding conic curves in the street) 